\title{
CHRISTIAN DELPORTE
}

\section{La société médiatique du $\mathrm{XIX}^{\mathrm{e}}$ siècle vue du $\mathrm{XX}^{\mathrm{e}}$ siècle}

J'avoue avoir été un peu troublé par le sujet que les organisateurs du colloque m'ont demandé de traiter - je le dis évidemment avec une pointe de malice -, et ce pour deux raisons qui me permettent d'entrer immédiatement dans le vif du sujet.

D'abord, mais ce n'est pas le plus important, l'histoire des médias se satisfait mal des cloisonnements académiques, ceux qui, traditionnellement, distinguent »dixneuviémistes « et »vingtiémistes«, abusivement fondés sur les ruptures, là où, souvent, dominent les continuités historiques. Je veux bien, pour satisfaire à l'exercice, revêtir l'habit du spécialiste du $\mathrm{XX}^{\mathrm{e}}$ siècle, mais cette opposition pose, en matière de médias, davantage de problèmes qu'elle n'en résout.

Ensuite, un tel sujet suppose que, au-delà des nuances à apporter, le $\mathrm{XIX}^{\mathrm{e}}$ siècle comporte, en matière médiatique, une unité telle qu'on puisse l'observer comme une période particulière, c'est-à-dire distincte de celle qui l'a précédée et de celle qui l'a suivie. La question des bornes chronologiques pour caractériser le $\mathrm{XIX}^{\mathrm{e}}$ siècle médiatique français fait débat chez les historiens. On peut légitimement s'interroger sur l'amont: que faire, ainsi, de l'héritage des Lumières et, plus encore, de la Révolution française? Le $\mathrm{XIX}^{\mathrm{e}}$ siècle médiatique commence-t-il dans les années 1770-1780, quand s'affirme l'espace public, quand se fait jour une opinion publique susceptible de critiquer ou de contrôler les actes du pouvoir monarchique? Doit-on plutôt le faire débuter en 1789, lorsque la question de la liberté d'expression s'installe au cœur du débat public? Ou, alors, faut-il porter le regard au temps de la monarchie de Juillet ${ }^{1}$, voire du Second Empire, quitte à le prolonger jusqu'à l'aube des années 1930, comme préconisent les historiens culturalistes? Car l'autre grande question est celle de l'aval: le Second Empire'2? l'instauration de la $\mathrm{III}^{\mathrm{e}}$ République et l'explosion de la presse qui suit la loi de 18813? la Belle Époque et son foisonnement médiatique4? la Grande Guerre et le point ultime de la conquête du public par la presse ${ }^{5}$ ? la fin des années 1920 et le basculement vers une culture audiovisuelle6? voire la veille de la Seconde Guerre mondiale?

1 Cf. Marie-Ève ThÉRENTY, Alain VaILlant, 1836, l'an I de l'ère médiatique. Étude littéraire et historique du journal »La Presse«, d'Émile de Girardin.

2 Cf. Marc Martin, Médias et journalistes de la République, Paris 1997.

3 Cf. Claude Bellanger et al. (dir.), Histoire générale de la presse française, vol. 3: De 1871 à 1914, Paris 1972; Agnès CHAUVEAU, Philippe TÉTART, Introduction à l'histoire des médias en France, de 1881 à nos jours, Paris 1999.

4 Cf. Laurent MARTin, La presse écrite en France au XX ${ }^{\mathrm{e}}$ siècle, Paris 2006.

5 Fabrice d'Almeida, Christian Delporte, Histoire des médias en France, de la Grande Guerre à nos jours, Paris 1999.

6 Cf. Dominique KaliFA, La culture de masse en France, vol. 1: 1860-1930, Paris, 2001.

7 Cf. Christophe ChARLE, Le siècle de la presse, 1830-1939, Paris 2004. 
Tout cela n'est pas simple. Mais le débat sur les bornes souligne d'abord la difficulté à concilier les »entrées«, les clés de compréhension pour saisir la nature, le rôle, l'importance des médias et les pratiques sociales qui en découlent. La question médiatique relève, en effet, de tous les grands champs historiques: histoire politique, histoire économique, histoire sociale, histoire culturelle. Les questionnements eux-mêmes ne se recoupent pas nécessairement. Longtemps, l'histoire politique a fixé les scansions de l'histoire médiatique; désormais, l'histoire culturelle a pris le pas sur la précédente pour proposer sa propre périodisation. Mais, derrière la périodisation même émerge l'interprétation globale. Ce jeu de balancier - il me semble - brouille, plus qu'il n'éclaire, la place des médias dans la société. Une approche trop politique a tendance à restreindre l'objet, à privilégier les projets au détriment des pratiques. Une approche trop culturaliste tend mécaniquement à gommer les particularités, voire les particularismes nationaux, qui permettent de saisir pourquoi, aujourd'hui encore, les médias français ne sont pas exactement identiques aux médias allemands, par exemple.

Si j'insiste sur ces aspects, c'est évidemment parce qu'ils orientent le regard sur les héritages du XIX ${ }^{\mathrm{e}}$ siècle, l'angle que je privilégierai ici, en évitant de m'enfermer dans une chronologie trop rigide. »Globalisant « par nature, mon propos n'évitera l'écueil du schématisme. Laissant de côté bien des éléments de contexte, il privilégiera, plus que le fonctionnement même de la société médiatique, les conditions qui le commandent.

Qu'est-ce que l'information? Les débats qui agitent la presse du $\mathrm{XX}^{\mathrm{e}}$ siècle autour de cette notion plongent leurs racines dans un large $\mathrm{XIX}^{\mathrm{e}}$ siècle marqué par l'affirmation d'un espace public libéral. Au fond, les questions qui se posent aujourd'hui dans la société française trouvent leur origine au siècle dernier, et notamment celle-ci, fondamentale: l'information est-elle un produit de consommation comme un autre? $\mathrm{Ce}$ qui m'amène à avancer deux mots clés, sur lesquels je vais fonder mon intervention, qui, à différents degrés, caractérisent le $\mathrm{XIX}^{\mathrm{e}}$ siècle et résonnent au cœur du $\mathrm{XX}^{\mathrm{e}}$ siècle: »éducation« et »marché«.

\section{LA MISSION ÉDUCATIVE DE LA PRESSE}

$\mathrm{Du}$ point de vue politique, on admet généralement que la question centrale du $\mathrm{XIX}^{\mathrm{e}}$ siècle est celle de la liberté d'expression, réprimée, contestée, conquise. C'est vrai, mais à condition de la lier à celle, tout aussi fondamentale, de la fonction sociale de la presse; autrement dit: à quoi la liberté de la presse doit-elle servir?

Depuis la Révolution française, et durant la plus grande partie du XIX ${ }^{\mathrm{e}}$ siècle, la conception de la presse est dominée par la notion large d'éducation et d'édification du plus grand nombre par les élites: élites sociales éclairées, riches du savoir universel, détentrices des visions d'avenir, ou élites conscientes, mues par la critique sociale et proposant une solution alternative à la société libérale. Libéraux et marxistes se rejoignent cependant. Pour les premiers, la presse est une œuvre civilisatrice, un outil de connaissance, de moralisation, d'émancipation, un instrument destiné à modeler l'opinion publique, c'est-à-dire à faire partager par le plus grand nombre les idées 
justes des élites. Pour les seconds, la presse est un moyen de prise de conscience collective des antagonismes sociaux, qui doit aboutir au renversement des élites sociales, et donc à la révolution. La presse doit »former et fortifier l'opinion«, écrit Benjamin Constant dans »La Minerve française«, en avril 1819. Elle est, pour Jules Guesde, une arme de combat (et donc de propagande) destinée à faire pénétrer les idées communistes dans les masses pour, finalement, les faire agir. Certes, la notion d'opinion s'oppose à la notion de classes et de masses; certes, l'acceptation ou le consensus s'opposent au refus, à l'action, à la révolution. Mais dans les deux cas, l'information ne saurait se satisfaire du »fait brut« - de la religion du »fait« -, quelle qu'en soit la catégorie, politique ou non. Le journaliste n'est donc pas un médiateur parmi d'autres, mais un missionnaire, un guide, un éducateur. Et c'est bien dans ce cadre que s'inscrit le projet de »presse à bon marché«. »La presse, écrit Girardin en $1836^{8}$, est, après l'instruction primaire, le moyen le plus actif de moralisation populaire«. Et les fondateurs du »Siècle« notent, lors de leur assemblée générale, en 1840:

Cette œuvre à laquelle nous nous sommes unis, c'est d'éducation morale et politique du peuple, son apprentissage de la liberté. Pour y réussir, il s'adressera à toutes les bourses, pour rendre notre auditoire le plus nombreux possible.

Pour les libéraux, mais aussi pour les marxistes, l'information relève d'un schéma vertical, où le journaliste parle et le lecteur écoute, le premier s'exprimant pour le plus grand bien du second. J'ajoute que le rôle »formateur « de la presse est un point de vue partagé par les catholiques, et qui justifie l'émergence de ce que ces derniers appellent la »bonne presse«, dont »La Croix«, quotidien lancé en 1883, est le plus digne représentant.

Ce projet libéral trouve son aboutissement avec la République et la loi sur la liberté de la presse de 1881. Le triomphe de la République et du suffrage universel imposent de donner au citoyen, hissé au rang d'acteur et de décideur politique, des chances égales dans l'ascension sociale, mais aussi dans l'accès à l'information, pour que le vote ne soit pas, comme on le dit alors, la »dictature des imbéciles«; une information destinée à hausser les capacités d'analyse du citoyen, mais aussi à fédérer l'opinion autour d'un projet commun. Du coup, la presse apparait, avec l'instruction généralisée, comme l'un des deux grands piliers de l'édification du peuple. Le journaliste, comme l'instituteur, reçoit une ımission` de salut public. La presse, et plus généralement les médias, remplissent, en effet, une véritable `mission « d'instruction civique, qui s'inscrit dans un programme idéologique fort. Les républicains ne doutent pas de leur réussite, ce qui explique le statut très libéral de la loi, sur le plan politique, mais aussi sur le plan économique. Les républicains sont majoritaires dans la presse, et la presse garantira l'achèvement de la conquête de l'opinion.

Cette conception de la presse comporte des effets multiples. Elle explique, par exemple, le poids et le statut des journaux dits »d'opinion« ou, comme on les nomme souvent au début de la $\mathrm{III}^{\mathrm{e}}$ République, les journaux »doctrinaux«, ce dernier mot ne

8 Dans le prospectus de lancement de son nouveau quotidien. 
comportant pas, comme à notre époque, pressée par les souvenirs douloureux des totalitarismes, une connotation dépréciative. Elle justifie aussi la présence dans des associations communes ${ }^{9}$, qui naissent dans les années 1880 et dessinent les contours d'une identité professionnelle, des patrons de presse et de leurs employés, les journalistes, unis par un idéal partagé, par une même mission ${ }^{10}$. Elle éclaire sur les jugements de valeur qui distinguent la »presse de qualité« de celle qui ne l'est pas. Elle fixe les domaines et les genres nobles du journalisme (dont le commentaire politique, l'éditorial, en est aujourd'hui l'héritier) comme les modèles d'écriture. Bref, elle établit une hiérarchie à laquelle se réfèrent les hommes du $\mathrm{XX}^{\mathrm{e}}$ siècle.

Pourtant, au moment même où la loi grave implicitement dans le marbre les repères idéalisés de la mission d'information, ceux-ci s'effacent ou se transforment. Derrière les critiques qui fusent, au tournant du siècle, contre la »nouvelle presse« s'expriment l'échec et l'amertume des élites. Le triomphe de la presse quotidienne à grand tirage bouleverse les équilibres et marginalise déjà les journaux d'opinion, dont la »crise de langueur« ne trouve pas ses origines dans les années 1950, comme on l'écrit souvent, mais déjà dans les années 1880-1890.

Les attaques se portent alors sur l'espace grandissant du roman-feuilleton, du fait divers, du sang ou du crime à la une ${ }^{11}$. On s'en prend à l'immoralité des journaux, aux facilités indignes du divertissement, à la dégradation de l'écriture et, finalement, à l'»américanisation« de la presse, perçue, en quelque sorte, comme le passage de la vulgarisation à la vulgarité. »Le journalisme abaisse tout, déforme tout, les hommes et les idées«, s’indigne Mirbeau dans »La France«, en 1885.

Sous prétexte de progrès, poursuit-il, et par une fausse, une maladroite application des procédés de vulgarisation rapide, il a pris au journalisme américain ce que celui-ci avait de plus mauvais, ayant soin de laisser de côté ce qu'il pouvait contenir de bon ${ }^{12}$.

Mais, derrière ce procès s'exprime surtout la crainte d'une société où les élites autoproclamées sont en train de perdre la main. Alfred Fouillée, l'un des plus virulents procureurs de la presse »nouvelle« écrit ainsi, en 1900, dans »La France du point de vue moral«: »Le problème qui se pose à notre démocratie est $[. .$.$] la reconquête de la$ presse par l'élite intellectuelle et morale«. Pourtant, quand on y regarde de plus près, la

9 Cf. MARTin, Médias et journalistes de la République (voir n. 2).

10 Cf. Christian Delporte, Les journalistes en France (1880-1950). Naissance et construction d'une profession, Paris 1999.

11 Cf. Lise DumASY (dir.), La querelle du roman-feuilleton. Littérature, presse et politique, un débat précurseur, 1836-1848, Grenoble 1999; Dominique KALIFA, L'encre et le sang. Récits de crimes et société à la Belle Époque, Paris 1995; Anne-Claude AMBROISE-RENDU, Petits récits des désordres ordinaires. Les faits divers dans la presse française des débuts de la $\mathrm{III}^{\mathrm{e}}$ République à la Grande Guerre, Paris 2004; Marine MsiLI, Le fait divers en République. Histoire sociale de 1870 à nos jours, Paris 2000.

12 Cf. Christian DelPORTE, L'américanisation de la presse? Éclairages sur un débat français et européen (années 1880-années 1930), dans: Jean-Yves MOLLIER, Jean-François SIRINELLI, François VALlOTTON (dir.), Culture de masse et culture médiatique en Europe et dans les Amériques, 1860-1940, Paris 2006, p. 209-222. 
nouvelle hiérarchie des genres journalistiques qui prend corps sous l'effet de la »révolution de l'information«, avec notamment l'apport du reportage, doit plus à la continuité ou à l'adaptation qu'à la rupture. L'art du récit du reporter se confond ainsi avec le vieil idéal d'écriture, et sa démarche même reste fidèle à l'idéal de mission confié au journaliste. Un bon reportage est un reportage édifiant. Le grand reporter, tout particulièrement, ne renonce jamais, dans ses articles, à vulgariser la connaissance; mais il fait davantage: fort des principes moraux qui fondent, plus qu'une fonction, une profession, il rapporte la »vérité« qui édifie l'opinion. D’une manière générale, du reste, et contrairement à ce que prétendent alors ses détracteurs, la presse »nouvelle«, loin de se situer dans la transgression, conforte le modèle social dominant et les valeurs sur lesquelles il repose, comme le confirment les études du discours sur le fait divers ${ }^{13}$.

Le $\mathrm{XX}^{\mathrm{e}}$ siècle hérite donc d'une conception de la presse qui s'explique par les conditions dans lesquelles s'est forgé l'espace public français, à savoir le lien organique liant l'information au pouvoir, la place de l'État dans le dispositif, le rôle particulier qu'y joue le modèle politique libéral, inspirateur de la République naissante. Il est d'ailleurs frappant de constater combien la mythologie de l'histoire de la presse au $\mathrm{XIX}^{\mathrm{e}}$ siècle (le récit héroïque de la conquête de la liberté) pèse sur les regards et les comportements des hommes de presse du $\mathrm{XX}^{\mathrm{e}}$ siècle; une mythologie qui domine toute la première moitié du siècle, jusqu'à ce qu'elle soit remplacée par une autre, celle de l'âge d'or de la Libération, refondatrice d'un modèle éducatif de l'information, porteur de valeurs morales professionnelles revivifiées.

\section{LES »LOIS DU MARCHÉ«}

Pourtant, l'héritage du $\mathrm{XIX}^{\mathrm{e}}$ siècle ne se limite pas au legs idéologique. Ce que les hommes de presse comprennent mal ou partiellement - pas tous, néanmoins -, c'est que le libéralisme politique suppose un libéralisme économique qui en brouille les repères. C'est là qu'intervient l'autre mot clé: le »marché«. Le XIX ${ }^{\mathrm{e}}$ siècle, en effet, est l'âge où s'affirme la consommation médiatique, où la presse est saisie par les lois du marché, jusqu'à paradoxalement placer en contradiction l'idéal du libéralisme politique et les logiques du libéralisme économique. Le lectorat des journaux n'est pas seulement un matériau modelable aux idées du temps; c'est aussi, et surtout, un marché où l'offre médiatique s'adapte à la demande sociale. On découvre alors peu à peu une règle commune à toute industrie culturelle: il n'y a pas de produit sans consommateur; il n'y a pas de journal sans lecteurs; la viabilité d'un journal dépend de sa capacité à répondre aux attentes, aux motivations, aux représentations du lecteur dans toute sa complexité sociale.

L'histoire des transformations de la presse au XIX ${ }^{\mathrm{e}}$ siècle, au fond, est l'histoire de l'élargissement toujours amplifié du lectorat, qui va à la rencontre de journaux répondant à ses aspirations et s'adaptant à son imaginaire; l'histoire de l'élargissement, mais aussi de la fidélisation. Car la conquête du lectorat populaire par la grande presse

13 Cf. Ambroise-Rendu, Les faits divers (voir n. 11). 
quotidienne ne s'explique pas seulement par des formats ou des contenus, mais aussi par une adaptation aux moyens et aux comportements des publics visés. J'évoque ici, bien sûr, la »révolution« des quotidiens à »un sou« vendus au numéro - qui s'engage avec l'apparition du »Petit Journal« en $1863^{14}$-, la rupture avec la vieille pratique de l'abonnement (bien plus précoce en France qu'en Allemagne, du reste), mais aussi la fixation des points de vente, élément indispensable pour fidéliser la clientèle.

La prise de conscience de l'existence d'un marché intervient au moment de la monarchie de Juillet, avec »La Presse« et »Le Siècle «15. Mais la difficulté de ces deux quotidiens à élargir le cercle de leur lectorat, à conquérir la frange des couches populaires cultivées, les marges de l'audience occasionnelle, souligne, selon moi, les limites de ce marché. En 1838, »Le Siècle« tire à 16000 exemplaires environ, »La Presse« à 13000 , à peine plus que »Le Journal des débats« $(10000)^{16}$. Dans les années 1840 , la totalité du tirage de la presse quotidienne n'excède pas 100000 à 150000 exemplaires. En 1845, »Le Siècle« en est à 35000 exemplaires, »La Presse« à 2200017 . Et l'écart avec la presse traditionnelle des élites pour les élites n'est pas considérable. La presse à »bon marché« joue sur les deux tableaux. Elle choisit un marché qu'elle surestime. Elle s'adapte, certes, aux attentes supposées de celui-ci, mais reste fondamentalement fidèle au modèle libéral de l'information.

L'échec des journaux de 1848 me semble également illustrer l'étroitesse du marché auquel ils s'adressent ${ }^{18}$. Ces journaux de propagande parlent au peuple et, par conséquent, pratiquent des tarifs de prix bas. Mais, à la fois, ils se montrent incapables de se faire entendre des couches populaires, et leur lectorat reste faible, alors que moins de la moitié des Français savent lire. Certes, les classes populaires parisiennes se sont familiarisées avec la lecture, mais les foules urbaines de province, et plus encore les populations rurales, restent à l'écart de l'alphabétisation. L'étape du »Petit Journal«, en 1863, est évidemment capitale. Toutefois, il est un peu l'arbre qui cache la forêt. Une fois la liberté acquise, d'autres titres tentent d'en adapter les recettes. Mais il faut attendre la fin des années 1880 et le début des années 1890 pour que des quotidiens comme »Le Petit Parisien«, »Le Matin« ou »Le Journal« trouvent enfin leur public.

En 1869, »Le Petit Journal« est le seul quotidien à plus de 100000 exemplaires $^{19}$. En 1880, on en compte cinq ${ }^{20}$, mais »Le Petit Journal« est unique en son genre, les autres restant des quotidiens politiques (comme »La Petite République« ou »La Lanterne $\ll$ ) ou des quotidiens des élites (comme »Le Figaro«). Près de trente ans plus tard, en 1908, on est passé à sept quotidiens de plus de 100000 exemplaires. Mais la diffé-

14 Cf. Marc MARTin, La réussite du »Petit Journal« ou les débuts du quotidien populaire, Bulletin du Centre d'histoire de la France contemporaine 3 (1982), p. 11-36.

15 Cf. VAillant, THÉRenTy (dir.), 1836 (voir n. 1).

16 Cf. Pierre Albert, Gilles Feyel, Jean-François PiCARD, Documents pour l'histoire de la presse nationale aux $\mathrm{XIX}^{\mathrm{e}}$ et $\mathrm{XX}^{\mathrm{e}}$ siècles, Paris 1976.

17 »Le Constitutionnel« est alors tiré à 23000 exemplaires.

18 Cf. Anne-Claude AmBroise-RENDU, Les journaux du printemps 1848: une révolution médiatique en trompe-1'œil, dans: Revue d'histoire du XIX ${ }^{\mathrm{e}}$ siècle 19 (1999), p. 35-64.

19154000 en octobre 1864; 259000 en décembre 1865; 340000 en novembre 1869.

20 Août 1880: »Le Petit Journal«, 583000 exemplaires; »La Petite République«, 196 000; »La Lanterne«, 150 000; »Le Petit Moniteur« et »Le Figaro« sont aux environs de 100000 exemplaires. 
rence, cette fois, c'est que les quatre premiers sont des quotidiens à un sou, dont le plus faible, »Le Matin«, tire à 620000 exemplaires, c'est-à-dire quatre fois plus que celui qui le suit, représentant de la presse traditionnelle des élites, »L'Écho de Paris«. C'est pourquoi la vraie conquête du public populaire par le quotidien - je dis bien le »quotidien« et non le »journal«, qui, sous diverses formes, le touche depuis longtemps - se situe des années 1880 à la Belle Époque. C'est là véritablement que s'affirme pour la presse l'âge de la consommation de masse.

Évoquer les années 1880 nous renvoie, une fois de plus, vers la République et la loi sur la liberté de la presse. Pourtant, ici, le facteur est au moins aussi social que politique. On peut, du reste, rapprocher cette époque d'une autre, celle de la Révolution française. On dit, et on a raison de l'affirmer, que 1789 est marqué par une explosion des journaux. Mais la liberté conquise aurait été de moindre effet pour la presse sans la préexistence d'un lectorat et d'habitudes de lecture, autrement dit sans la réactivité ou la réceptivité de couches instruites ${ }^{21}$. En 1789, l'offre médiatique répond à une demande sociale; au début de la III ${ }^{\mathrm{e}}$ République, il en va de même. La liberté est, certes, un levier important du développement de la presse. Mais tout aussi capital est le marché potentiel formé en amont à la lecture (y compris de la »petite presse«), grâce aux lois scolaires, de Guizot (1833) à Duruy (1867), de telle sorte que, sous le Second Empire, la quasi-totalité des communes dispose d'une école et que l'analphabétisme, en 1880 , est tombé à $17 \%$ de la population. Dans ces conditions, le marché ne demande qu'à être libéré: c'est ce qui se passe en 1789 comme en 1881, dans des proportions évidemment inégales ${ }^{22}$.

\section{»LE LECTEUR A TOUJOURS RAISON«}

Certes, les quotidiens de masse puisent leur succès dans l'adaptation aux attentes d'un lectorat populaire. Et on en voit le signe dans l'essor de genres particuliers, comme le fait divers et le récit aux ressorts émotionnels, ou dans le primat donné aux nouvelles sensationnelles, etc. Mais, à mon avis, on néglige beaucoup trop la nature même de l'information, c'est-à-dire le lien entretenu par le lecteur avec l'information lue dans le journal. Et, à cet égard, je prendrai deux exemples aux fortes résonances contemporaines.

Le premier tient à l'écriture de presse elle-même. »Faites simple, faites court, faites accessible«, recommande-t-on aux journalistes, aujourd'hui. Or, c'est bien là un des secrets de quotidiens comme »Le Petit Journal«. Très caractéristique à cet égard, une pétition se distingue, signée par 43 ouvriers fondeurs en 1868, adressée aux députés, et qui s'oppose au projet de soumettre la presse à un sou au droit de timbre, pour rééquilibrer la concurrence avec les quotidiens politiques. Des ouvriers »instruits« défendent

${ }_{21}$ Cf. Gilles FeYEL, L'annonce et la nouvelle. La presse d'information en France sous l'Ancien Régime, 1630-1788, Oxford 1999.

22 En Allemagne, l'alphabétisation est plus précoce qu'en France. Mais les journaux, dans les années 1880-1890, restent réservés aux élites, pour l'essentiel, et continuent à être distribués par abonnement. Vissés par le pouvoir, ils demeurent très »idéologiques«, et finalement peu ouverts sur le marché. 
»Le Petit Journal«. Ils expliquent qu'ils en apprécient les genres (les romans, les fictions), mais aussi la façon d'écrire. En gros, disent-ils, »Le Petit Journal « parle comme nous, pour nous, contrairement aux journaux politiques, qui, eux, usent de $»$ mots inconnus à chaque ligne « ${ }^{23}$. Un »journaliste du peuple« comme Jules Vallès a bien compris l'engouement populaire pour un tel quotidien. Et loin de s'offusquer et de rejoindre la meute méprisante des élites bourgeoises ou ouvrières, il écrit dans »Le Figaro«, dès le 21 janvier 1866, à propos de la presse d'opinion:

Elle n'a pas voulu, l'orgueilleuse, quitter la toge et le manteau pour prendre cette allure légère et vive qui plaît à un peuple las des tirades et que les grands mots et les longues tartines n'ont pas sauvé de la misère et ont mené quelques fois à l'abattoir. Tous [...], nous devons compter avec l'esprit populaire du temps et nous rappeler que nous parlons à une société nouvelle, à laquelle il faut un art nouveau.

L'adaptation que Vallès appelle de ses vœux correspond à une lecture finalement juste d'une société des années 1860 en pleine mutation. Au-delà, c'est un appel à l'adaptation permanente de la presse aux mutations de la société que le $\mathrm{XX}^{\mathrm{e}}$ siècle, lui, finit par admettre, pour ne pas perdre le contact avec le lecteur.

Le second exemple se situe sur un autre plan. L'expansion des grands quotidiens populaires, et particulièrement d'un journal comme »Le Petit Parisien«, bientôt le premier quotidien au monde par le tirage ${ }^{24}$, tient d'abord à leur capacité de conquérir le public de province. La constitution d'un réseau de diffusion est évidemment fondamentale, et par ailleurs bien connue. Toutefois, il me semble important de souligner que le quotidien parisien a su concilier, grâce à l'essor des agences, la nouvelle nationale ou internationale la plus »fraîche«, si j'ose dire, et l'information de proximité de qualité, grâce à son réseau de correspondants locaux. Cette loi de la proximité est inchangée de nos jours. Dans une information mondialisée, elle a même tendance à se renforcer. Le lecteur est d'abord intéressé par l'information qui le touche directement: ce qui se passe dans son immeuble, son quartier, son village compte toujours plus que ce qui se déroule à l'autre bout du monde, sauf si l'événement peut avoir pour lui des conséquences concrètes. C'est sur ce fondement que, à partir des années 1880-1890, les quotidiens de province commencent leur expansion ${ }^{25}$. Grâce à leurs éditions départementales et à leur réseau serré de correspondants, ils finissent, au début du $\mathrm{XX}^{\mathrm{e}}$ siècle, par damer le pion à la presse parisienne. Cette recette de la proximité guide encore la réussite des médias aujourd'hui et explique, en partie au moins, la relative résistance des quotidiens régionaux au déclin de la presse quotidienne. Bref, l'héritage direct du journal tel qu'il a pu se transformer au XIX ${ }^{\mathrm{e}}$ siècle est une conception de l'information qui ouvre deux fenêtres à son lecteur: l'une sur le monde, l'autre sur son jardin.

${ }^{23}$ Cf. Élisabeth CAZENAVE, Fonctions identitaire et émancipatrice de la presse des classes populaires au XIX ${ }^{\mathrm{e}}$ siècle, dans: Roger BAUTIER, Élisabeth CAZENAVE, Michael PALMER (dir.), La presse selon le XIX ${ }^{\mathrm{e}}$ siècle, universités de Paris III et Paris XIII 1997, p. 27-37.

24 Cf. Francine Amaury, Histoire du plus grand quotidien de la III ${ }^{\mathrm{e}}$ République: »Le Petit Parisien«, 1876-1944, Paris 1972.

25 Cf. Marc MARTin, La presse régionale. Des affiches aux grands quotidiens, Paris 2002. 


\section{UN PUBLIC? DES PUBLICS!}

Il y aurait encore beaucoup à dire sur les héritages du XIX ${ }^{\mathrm{e}}$ siècle. Je n'insisterai pas sur les transformations du journalisme et l'émergence de la profession de journaliste sur lesquelles je me suis déjà abondamment exprimé26. Je voudrais juste, pour finir, évoquer brièvement quelques points caractéristiques de la plongée de la société médiatique du XIX ${ }^{\mathrm{e}}$ siècle dans le siècle suivant.

Il conviendrait ainsi d'insister sur l'élargissement des outils médiatiques, et singulièrement les supports visuels, comme la photographie, le cinématographe, bien sûr, mais aussi l'affiche publicitaire, dont je rappellerai le statut et la légitimité que lui accordent les Expositions universelles de Paris en 1889 et en 1900, comme signe fort de la modernité française. À travers la diversité des messages, codifiés par les mots et les images, se dégagent des valeurs soigneusement pensées en fonction des groupes sociaux ciblés par la publicité27. La prise en compte de la diversité des publics, en effet, est une donnée essentielle du développement médiatique, dès lors aussi que l'espace de communication repose sur un maillage serré, qu'il couvre le territoire, la ville, le quartier, dès lors que l'achat du journal en des lieux rigoureusement fixés (le kiosque, la bibliothèque de gare ${ }^{28}$, notamment) relève du réflexe quotidien, bref, dès lors que s'est installée une habitude sociale.

Cette prise en compte de la diversité des attentes selon la situation sociale - clé de compréhension de l'offre médiatique du $\mathrm{XX}^{\mathrm{e}}$ siècle - joue sur la verticalité des groupes sociaux (élites et masses), mais aussi sur l'horizontalité d'un même groupe social (les hommes, les femmes, les enfants). Il suffit de voir les contenus des suppléments illustrés des grands quotidiens de la fin du XIX ${ }^{\mathrm{e}}$ siècle, qui, au fil des pages, s'adressent aux différentes composantes du foyer: le chef de famille, l'épouse, la mère, la ménagère, les enfants aussi. Le plus caractéristique, à cet égard, est l'essor de la presse hebdomadaire spécialisée, comme le montre le cas de la presse féminine, avec, à côté des journaux s'adressant traditionnellement aux femmes de la bonne société, la percée du »Petit Écho de la mode«, visant les ménagères des catégories moyennes et adaptant son prix et ses contenus à leurs attentes. Rappelons que l'hebdomadaire, lancé en 1878, voit son tirage tripler entre 1885 et 1900, passant de 100000 à 300000 exemplaires.

La question de l'image, suggérée à propos de la publicité, mériterait aussi d'amples développements. Un exemple intéressant, à cet égard, est la transformation du marché de la vieille imagerie d'Épinal, qui sauve provisoirement son existence en s'adaptant aux nouvelles conditions de la société médiatique. D'abord, l'imagerie Pellerin, dans

${ }^{26}$ Cf. DELPORTE, Les journalistes en France (voir n. 10).

27 Cf. notamment: Réjane BARgIEL-HARRY, Christophe ZAgrodzKi, Le livre de l'affiche, Paris 1985; Alain WeILl, L'affiche dans le monde, Paris 1991 (rééd.); Christian DelPORTE, De Bibendum à Culture pub, dans: Jean-Pierre RIOUX, Jean-François SIRINELLI (dir.), La culture de masse en France de la Belle Époque à nos jours, Paris 2002, p. 410-434.

28 Cf. Karine TAVEAUX-GrandPIERRE, De la diffusion de la presse parisienne quotidienne en France: Hachette et les quotidiens à grand tirage, 1870-1914, thèse de doctorat en information et communication, université de Paris III 1999; Gilles FEYEL (dir.), La distribution et la diffusion de la presse, du XVIII ${ }^{\mathrm{e}}$ siècle au $\mathrm{III}^{\mathrm{e}}$ millénaire, Paris 2002. 
les années 1890 , prend acte de l'inadaptation du colportage par rapport au marché. Le colporteur se transforme peu à peu en représentant de commerce auprès des grands magasins (La Belle Jardinière, Aux Trois Quartiers...) et des grandes entreprises (alimentation, confiserie, pharmacie, habillement...), et la part des libraires dans le chiffre d'affaires augmente lentement à partir des années 1880 (39\% en 1883; 45\% au début du $\mathrm{XX}^{\mathrm{e}}$ siècle). Et puis, Pellerin réduit la gamme de sa production, d'abord en transformant l'image, naguère dominée par les thèmes religieux, en supports publicitaires, ensuite en ciblant la clientèle des enfants appartenant aux groupes sociaux aisés et citadins, comme l'indiquent les planches d'historiettes ou les jeux à découper ${ }^{29}$.

L'image, support essentiel dans le mouvement de démocratisation médiatique du $\mathrm{XX}^{\mathrm{e}}$ siècle, reste encore à la fin du $\mathrm{XIX}^{\mathrm{e}}$ siècle un objet de luxe, comme l'indique le cas de la photographie. Les magazines illustrés, de »L'Illustration« à »Fémina«, ne s'adressent pas au plus grand nombre. À une certaine conception du rôle de la photographie considérée comme un art (»L'Illustration« ne l'adopte que dans les années $1890^{30}$ ) s'ajoute le coût de reproduction, qui pèse sur le prix de vente et restreint, de facto, la clientèle. Les tentatives pour imposer des quotidiens illustrés sur le modèle américain, dans les années 1880-1890 (avec »La Journée«, »L'Actualité«, »Le Quotidien illustré«, etc.), échouent. Ces échecs commerciaux s'expliquent par de multiples facteurs, techniques, économiques, financiers, mais aussi par un certain regard social (bref, un »état« du marché). Les quotidiens illustrés sont faits pour »les gens qui ne savent pas lire« pensent les élites, qui se détournent de journaux pourtant conçus pour elles. Il faut attendre 1912 et »Le Miroir«, et plus encore l'après-guerre, pour que, à l'imitation du cinéma, devenu un art et un moyen d'information populaires, la photographie, désormais facile à reproduire pour un coût réduit, garantisse la réussite de la grande presse populaire.

On le voit, la société médiatique qui caractérise le $\mathrm{XX}^{\mathrm{e}}$ siècle trouve largement ses racines dans le siècle précédent. À bien des égards, du reste, les mutations technologiques, économiques, culturelles qui affectent le système médiatique depuis vingt ou trente ans sont assez comparables à celles qui s'opérèrent à la fin du XIX $\mathrm{X}^{\mathrm{e}}$ siècle. La fin d'un monde pour la venue d'un monde nouveau inquiète ou enthousiasme, en tout cas suscite débat sur le sens et les effets de la rupture, nourrit des interrogations et des inquiétudes. Les hommes de presse de la fin du XIX $\mathrm{X}^{\mathrm{e}}$ siècle sentaient bien un mouvement qu'ils ne pouvaient arrêter, sans pouvoir en saisir toutes les dimensions. Aujourd'hui, les tensions perceptibles dans le monde médiatique provoquent de lourdes interrogations sur l'avenir: que va devenir la presse écrite? Quel avenir pour la »presse de qualité «? Que restera-t-il du pluralisme des quotidiens? Quelle place pour le journaliste-médiateur? Faut-il encadrer la concentration médiatique?, etc. Autant de questions qui renvoient à la situation du $\mathrm{XIX}^{\mathrm{e}}$ siècle, au temps où le rôle sacré de la presse semblait submergé par les logiques du marché, où la transformation des outils paraissait

29 Cf. Annie Delporte, L'imagerie d'Épinal, mémoire de DEA, université de Lille III 1994.

$30 \mathrm{Cf}$. Anne-Claude AmBroise-Rendu, Du dessin de presse à la photographie (1878-1914): histoire d'une mutation technique et culturelle, dans: Revue d'histoire moderne et contemporaine 39 (1992), p. 6-28. 
emporter les valeurs de la presse, soumise à une dégradation mortelle ${ }^{31}$. À l'époque, la prise en compte du marché avait permis la démocratisation de la lecture, tout en suscitant, en filigrane, une question non résolue de nos jours: la démocratisation médiatique sans contrôle sert-elle, oui ou non, la démocratie?

31 Maret, en 1891, évoquait ainsi les transformations du »quatrième pouvoir«, en introduction de l'Annuaire de la presse: »Plus vite, toujours plus vite. Ce fut dans les journaux une lutte de rapidité, comme aux courses. Le public ne voulut plus attendre pour être informé de tout, des petites choses plus que des grandes«. Et de préciser: »Le bulletin bibliographique et l'interview remplacèrent l'étude littéraire. Au théâtre, plus de feuilleton, on voulut savoir dès le lendemain ce qui s'était passé [...], de là des comptes rendus improvisés, sans méthode, sans esthétisme, au hasard de la fourchette«. 
